\title{
Determining Content Validity of A Clinical Reasoning Educational Intervention Package Using An Expert Panel
}

\author{
${ }^{1}$ YAURI, Indriani; ${ }^{2} \mathrm{NASH}$, Robyn; ${ }^{3}$ RAMSBOTHAM, Joanne \\ ${ }^{1}$ Faculty of Nursing Catholic University of De La Salle Manado, Indonesia; ${ }^{2.3}$ School of \\ Nursing, Faculty of Health Queensland University of Technology, Australia
}

\begin{abstract}
Background: Utilizing expert panel in content validity evaluations of new constructed educational packages in the nursing context have not been described well.

Objective: to describe the use of expert panel in a content validity evaluation of a new clinical reasoning educational package.

Methods: Five senior nursing academics participated in the study. The prospective panellists were selected based on their relevant professional expertise, including teaching experience, relevant academic qualifications, and practice experience in the Indonesian context. The specifically constructed feedback instrument consisted of 31 items encompassing an overview of the educational-intervention model, the student's workbook and the teacher's guide. A Likert scale was used to evaluate the relevance, clarity and feasibility of the educational intervention package. The review was conducted over a period of approximately five weeks. Numerical data were analysed using the Content Validity Index (CVI) method. Both Item (I-CVI) and Scale (S-CVI) levels were calculated. An I-CVI of $\geq .78$ and an S-CVI of $\geq .90$ are determined. Panellists' written comments were also sought. Thematic analysis was undertaken to analyse the qualitative data. Findings were used to inform decisions about whether to retain, modify or eliminate each item.

Results: The I-CVI scores for each sub-scale (relevance, clarity and feasibility) was $\geq .93$.

The S-CVI for the educational overview, teacher and student's guides was $\geq .95$. Accordingly, the average proportion of expert agreement on the educational-intervention package was .98. The package as a whole (and the three individual components: overview, teacher's and student's guides) was shown to have excellent content validity. Comments from the panellists are focus on the teacher's preparation before using the educational package. Thus, no further improvement on the educational package was required.

Conclusion: Utilizing expert panel content validity and critically evaluating their recommendations can result in a more relevant, clear and feasible educational intervention, leading for a more effective clinical reasoning educational approach for student nurses. Acknowledgment: This study was supported by Indonesian Higher Education Department and Queensland University of Technology, Australia
\end{abstract}

Keywords: expert panel, clinical reasoning, educational intervention package 